\title{
Prevalence and echo features of mediastinal lymph nodes in EUS for non-malignant indications: a prospective study in a Southern European Population
}

\section{(ㄷ)(i) $\Theta$}

\author{
Authors \\ Joana Carmo, Miguel Bispo, Susana Marques, Cristina Chagas
}

Institution

Gastroenterology Department, Hospital de Egas Moniz Centro Hospitalar de Lisboa Ocidental, Lisboa, Portugal

submitted 8.9.2017

accepted after revision $\quad 27.12 .2017$

\author{
Bibliography \\ DOI https://doi.org/10.1055/s-0044-101602 | \\ Endoscopy International Open 2018; 06: E432-E436 \\ (c) Georg Thieme Verlag KG Stuttgart · New York \\ ISSN 2364-3722
}

Corresponding author

Joana Carmo, Gastroenterology Department, Hospital Egas

Moniz - CHLO, Rua da Junqueira 126, 1349-019 Lisboa,

Portugal

Fax: +351213624139

joanavcarmo@gmail.com

\section{ABSTRACT}

Background and study aims Significant heterogeneity in geographic distribution regarding the prevalence of mediastinal lymph nodes (MLN) has been documented in autopsy and computed tomography (CT) studies. Awareness of the local prevalence and characteristics of lymph nodes will be relevant when performing endoscopic ultrasonography (EUS) for staging of malignant neoplasias. The aims of this study were to document the prevalence and echo features of MLN in patients undergoing EUS for non-malignant extrathoracic disease and to identify predictive factors for the presence of MLN.

Patients and methods A prospective single-center study was performed over 6 months. Mediastinal stations 9, 8, 7, $6,5,4 \mathrm{~L}$ and 2 were systematically evaluated using a linear echoendoscope in all patients undergoing EUS due to benign extrathoracic pathology and without history of oncologic disease. Demographic, clinical and EUS features of the lymph nodes were analysed.

Results Seventy-five patients were included: male/female 32/43; mean age, 63 years. The majority of patients ( $72 \%$ ) had lymph nodes in at least one mediastinal station and $88 \%$ of these were found in stations 7 or 4 L. Overall, 133 MLN were identified: $19 \%$ were hypoechogenic, $6 \%$ had a short-axis diameter $>10 \mathrm{~mm}$, and $6 \%$ were round. The prevalence of lymph nodes was higher in smokers ( $83 \%$ vs $64 \%$, $P=0.024)$, with a higher average number of lymph nodes per patient in this group (2.1 vs $1.6 ; P=0.017$ ). By logistic regression analysis, none of the variables analyzed were independently associated with the presence of MLN.

Conclusion This prospective Portuguese study documented a higher prevalence of MLN than previously reported in Northern Europe, in patients with no evidence of oncologic disease. This higher prevalence may negatively influence the specificity and positive predictive value for malignancy of MLN (N) staging by EUS.

\section{Introduction}

The map of mediastinal and pulmonary lymph nodes, described by Clifton F. Mountain and Carolyn M. Dresler in 1997, defines 14 intrathoracic lymph node stations: 1-9 mediastinal stations, $10-11$ hilar stations and $12-14$ intrapulmonary stations [1]. It has been extensively used since then, providing uniform, consistent and reproducible criteria to describe the position of every intrathoracic lymph node [1,2]. Thus, it has allowed the standardization of nodal staging in lung and upper gastrointestinal malignancies [2].
Today, endoscopic ultrasonography (EUS) is widely used for staging of these tumors [3]. In the staging of upper gastrointestinal malignancies (esophagus and stomach), EUS is crucial for providing not only accurate $\mathrm{N}$ staging but also T staging $[4,5]$. In non-small-cell lung cancer, EUS is a reliable staging modality and reduces both the number of mediastinoscopies and unnecessary thoracotomies $[3,6,7]$. Accuracy of EUS significantly improves when fine-needle aspiration (FNA) of the suspected lymph node is added [3]. Thus, EUS is of paramount importance in management and treatment planning for patients with these malignancies. 
EUS is an appropriate modality for assessing lymph nodes that lie adjacent to the esophagus in the following mediastinal stations: 4L/5 (aortopulmonary window), 7 (subcarinal space), 8 (lower paraesophageal) and 9 (pulmonary ligamentum) [3]. Stations 1, 2, 3 and $4 R$ are not always assessable, due to the air in the larger airways [3].

Previously described lymph node echo features suggestive of malignancy include hypoechoic echogenicity, sharp edges, round shape and a long-axis diameter exceeding $10 \mathrm{~mm}$ [8]. None of these EUS features alone was found sufficient to differentiate benign from malignant lymph nodes [9]. However, when all four of the above features are present in the same lymph node, the accuracy for predicting malignant invasion is $80 \%$ to $100 \%$ [8 - 10]. FNA improves EUS accuracy for diagnosis of malignancy, compared with lymph node echo features alone (sensitivity, $85-98 \%$; specificity, $100 \%$ ) [9-11]. However, FNA of lymph nodes is not always achievable (for example, because of primary tumor interposition in esophageal cancer staging).

Enlarged mediastinal lymph nodes and, eventually, false-positive results in EUS nodal staging, can be influenced by differences in geographic prevalence of various inflammatory diseases evolving mediastinal lymph nodes, such as sarcoidosis, histoplasmosis and anthracosilicosis.[3] Autopsy and CT studies from different countries have shown significant heterogeneity regarding prevalence and features of mediastinal lymph nodes [12-14]. The only two studies based on EUS which analyzed mediastinal lymph node burden in populations without underlying malignant disease showed a marked variation among United States (Indiana) and Northern European populations (Sweden and UK) $[15,16]$.

The aims of this study were to prospectively evaluate the prevalence and echo features of mediastinal lymph nodes in a Southern European population undergoing EUS for non-malignant extrathoracic disease and to identify predictive factors for the presence of mediastinal lymph nodes.

\section{Patients and methods}

All patients who were referred for EUS at the Endoscopic Unit of Egas Moniz Hospital, Lisbon, Portugal, due to non-malignant extrathoracic disease were evaluated. Patients with current or previous malignant disease were excluded. All patients were clinically evaluated in the previous 3 months and all had at least one imaging study (abdominal ultrasound, CT or magnetic resonance imaging) during this period. Seventy-five patients agreed to participate and were prospectively enrolled with informed consent. Sixty-eight patients underwent EUS for benign biliopancreatic disease and 7 for benign subepithelial lesions of the upper digestive tract (1 esophageal duplication cyst, 2 esophageal leiomyomas, 2 ectopic pancreatic rests and 2 gastric gastrointestinal stromal tumors $<3 \mathrm{~cm}$ ). The study was approved by the ethics committee.

All patients were evaluated by an experienced endosonographer (MB), using a linear echoendoscope (Pentax EG-3270UK), under conscious sedation (intravenous midazolam and fentanyl). At the time of the examination, after the abdominal EUS evaluation was complete, the scope was placed over the lower esophageal sphincter and mediastinal stations 9, 8, 7, 6, 5, 4L and 2 were systematically observed.

Demographic data (age, gender, race), clinical history (comorbidities; smoking habits; occupational exposure to potential causative agents of pneumoconioses - silica, asbestos or coal dust; and EUS indication and findings) were analyzed. Echo features of the lymph nodes were collected regarding location (mediastinal station, according to Mountain-Dresler classification [1]), size (short and long-axis diameters), shape (round, triangular/crescent or oval/ellipsoid), echo pattern [homogeneous echographic appearance - hypoechogenic, isoechogenic or hyperechogenic (using the adjacent mediastinal fat as a reference) versus central hyperechogenicity with more hypoechoic periphery], borders (sharp or indistinct) and presence of calcifications.

According to the EUS findings, on a case-by-case-based decision, follow-up with thoracic CT was recommended.

Data are expressed as average and minimum and maximum values. For comparative analysis, parametric (Student's $t$-test, for numeric variables) and non-parametric tests (Chi-Square and Mann-Whitney tests, for categorical variables and whenever normality could not be found) were used. Comparison between variables with a small sample size $(n<10)$ was not considered. To establish dependence between variables, logistic regression analysis was performed (backward-LR), considering the presence of mediastinal lymph nodes on EUS as the dependent variable. A $P$ value $<0.05$ was considered statistically significant. The statistical program used was SPSS for Windows, version 20.0 (Chicago, Illinois, United States).

\section{Results}

Seventy-five patients with a mean age of 63 years (range, 20 89 ) were enrolled in this study. Demographic and clinical data are shown in $>$ Table 1 . Twenty-eight percent of patients $(n=$ 21) had no identifiable mediastinal lymph nodes on EUS. Seven-

- Table 1 Patient demographic and clinical data.

\begin{tabular}{|l|l|}
\hline & n \\
\hline Gender (M/F) & $32 / 43$ \\
\hline Race (Caucasian/African natives) & $70 / 5$ \\
\hline Smoking habits (non/previous/current smoker) & $45 / 14 / 16$ \\
\hline Occupational respiratory exposure & 6 \\
\hline - Asbestos & 3 \\
\hline - Silica & 2 \\
\hline - Coal dust & 1 \\
\hline Indication for EUS & 49 \\
\hline - Suspected choledocholithiasis & 19 \\
\hline - Acute pancreatitis of unknown origin & 7 \\
\hline - Subepithelial lesion & \\
\hline EUS, endoscopic ultrasound & \\
\hline
\end{tabular}


- Table 2 Echo features of the detected mediastinal lymph nodes.

n

\begin{tabular}{|c|c|}
\hline & n \\
\hline \multicolumn{2}{|l|}{ Size } \\
\hline \multicolumn{2}{|l|}{ - Long axis } \\
\hline - Mean size \pm standard deviation (mm) & $10 \pm 4.8$ \\
\hline - Range (mm) & $3-29$ \\
\hline . $>10 \mathrm{~mm}$ & $63(47 \%)$ \\
\hline . $>20 \mathrm{~mm}$ & $19(14 \%)$ \\
\hline \multicolumn{2}{|l|}{ - Short axis } \\
\hline - Mean size \pm standard deviation (mm) & $5 \pm 2.5$ \\
\hline - Range (mm) & $2-18$ \\
\hline . $>5 \mathrm{~mm}$ & $38(29 \%)$ \\
\hline . $>10 \mathrm{~mm}$ & $8(6 \%)$ \\
\hline \multicolumn{2}{|l|}{ Shape } \\
\hline - Round & $8(6 \%)$ \\
\hline - Triangular/crescent & $46(35 \%)$ \\
\hline - Oval/ellipsoid & $79(59 \%)$ \\
\hline \multicolumn{2}{|l|}{ Echo pattern } \\
\hline - Homogeneous & $80(60 \%)$ \\
\hline - Hypoechogenic & 25 \\
\hline - Isoechogenic & 55 \\
\hline - Hyperechogenic & 0 \\
\hline - Central hyperechogenicity & $53(40 \%)$ \\
\hline \multicolumn{2}{|l|}{ Borders } \\
\hline - Sharp & 0 \\
\hline - Indistinct & $133(100 \%)$ \\
\hline Calcifications & $4(3 \%)$ \\
\hline
\end{tabular}

ty-two percent of patients $(n=54)$ had at least one mediastinal lymph node and more than half $(57 \%, n=43)$ had at least two mediastinal lymph nodes-mean number of lymph nodes per patient, $1.8 \pm 1.5$ (range, $0-6$ ).

Overall, 133 lymph nodes were detected, $88 \%$ in stations 7 or $4 \mathrm{~L}$ (station $7, n=71$; station $4 L, n=46$ ). In the subcarinal space (station 7), lymph nodes were larger than in $4 \mathrm{~L}$ station (long-axis diameter: 15 versus $8 \mathrm{~mm}$; short-axis diameter: 6 versus $4 \mathrm{~mm}$ ). Echo features of the detected lymph nodes are described in $>$ Table 2 .

Approximately half of the lymph nodes had a long-axis diameter larger than $1 \mathrm{~cm}(47 \%, n=63), 19 \%$ were hypoechogenic homogeneous, $6 \%$ were round and none had sharp borders. More than half of the lymph nodes $(56 \%, n=74)$ had at least one feature suggestive of malignancy [8] (most frequently, long-axis diameter larger than $1 \mathrm{~cm}$ ), $8 \%$ had two features suggestive of malignancy (long-axis diameter larger than $1 \mathrm{~cm}$ plus hypoechogenic homogeneous pattern or round shape) and none had three or four of these features. Analyzing the lymph node echo features per patient, more than half of patients ( $55 \%, n=41$ ) had lymph nodes larger than $1 \mathrm{~cm}$, approximately a quarter $(24 \%, n=18)$ had lymph nodes with a hypoechogenic homogeneous pattern and $11 \%(n=8)$ had lymph nodes with a round shape.

No differences were found between patients younger or older than age 65 regarding prevalence of mediastinal lymph nodes, the number of lymph nodes per patient or their echo features ( $\triangleright$ Table 3 ). Smokers (current or previous) had a higher prevalence of mediastinal lymph nodes ( 83 versus $64 \%, P=$ $0.024)$ and a higher number of lymph nodes per patient than non-smokers (2.1 versus 1.6, $P=0.017)$ ( $\triangleright$ Table 4$)$. Mediastinal lymph nodes in smokers more often had central hyperechogenicity with hypoechoic periphery than a homogeneous echo pattern ( $\triangleright$ Table 4$)$. Patients with significant respiratory occupational exposure had a higher prevalence of mediastinal lymph nodes and there was a higher number of lymph nodes per patient. However, due to the small number of patients in this group $(n<10)$, comparative analysis was not considered.

By logistic regression analysis, none of the analyzed demographic or clinical variables were independently associated with the presence of mediastinal lymph nodes on EUS.

Based on EUS findings, clinical evaluation and follow-up thoracic CT was recommended within 3 months in $9.3 \%$ of patients $(n=7)$, including patients with two lymph node features suggestive of malignancy and in the presence of lymph nodes with short-axis diameter $>1 \mathrm{~cm}$. All those patients remained asymptomatic and thoracic CT showed no significant dimensional changes of the lymph nodes.

\section{Discussion}

In this study, we prospectively examined 75 patients who underwent EUS for non-malignant disease, looking for mediastinal lymph nodes. We found a prevalence of mediastinal lymph nodes of $72 \%$ (54/75), higher than the one reported in Northern Europe by Kalaitzakis et al. [15] and lower than that documented in Indianapolis, United States, by Wiersema et al.[16]. We hypothesized that this could be related to the higher prevalence of tuberculosis in Southern Europe versus Northern Europe [17]. However, only two of our patients had a known history of tuberculosis (>10 years before EUS). The higher prevalence found in the United States could be related to the high prevalence of histoplasmosis, as stated by Wiersema et al. [16]. This is the most common mycosis in the United States and it is endemic in Indianapolis, Indiana[18]. The mean number of mediastinal lymph nodes per patient was also higher in the United States $(3.6 \pm 2.8$, range $0-14)$ than in Portugal ( 1.8 \pm 1.5 , range $0-6)$. Northern Europe had the lowest mean number of mediastinal lymph nodes per patient $(1.1 \pm 1.3$, range 0 $8)$. To our knowledge, these are the only three studies performed using EUS to analyze mediastinal lymph nodes in patients without malignant disease. According to the results of these studies, the distinct prevalence of different granulomatous diseases seems to influence the mediastinal lymph node background in different populations. Awareness of the local 
- Table 3 Comparison of the number of mediastinal lymph nodes and their echo features in patients $<65$ versus $\geq 65$ years.

\begin{tabular}{|c|c|c|c|}
\hline & $<65$ years $(n=40)$ & $\geq 65$ years $(n=35)$ & $P$ \\
\hline Prevalence of mediastinal lymph nodes & $30(75 \%)$ & $24(69 \%)$ & NS \\
\hline Average number of lymph nodes per patient & $1.9(78 / 40)$ & $1.6(55 / 35)$ & NS \\
\hline Long axis (mean) & $12 \mathrm{~mm}$ & $12 \mathrm{~mm}$ & NS \\
\hline Short axis (mean) & $5 \mathrm{~mm}$ & $4 \mathrm{~mm}$ & NS \\
\hline \multicolumn{4}{|l|}{ Largest mediastinal lymph node } \\
\hline " Long axis (mean) & $17 \mathrm{~mm}$ & $16 \mathrm{~mm}$ & NS \\
\hline - Short axis (mean) & $6 \mathrm{~mm}$ & $6 \mathrm{~mm}$ & NS \\
\hline Central hyperechogenicity $(n=53)$ & $42.3 \%(33 / 78)$ & $36.3 \%(20 / 55)$ & NS \\
\hline Round shape & $9(12 \%)$ & $1(2 \%)$ & NS \\
\hline
\end{tabular}

- Table 4 Comparison of the number of mediastinal lymph nodes and their echo features in smokers versus non-smokers.

\begin{tabular}{|l|c|c|}
\hline & Smokers (n=30) & Non-smokers(n=45) \\
\hline Prevalence of mediastinal lymph nodes & $25(83 \%)$ & $29(64 \%)$ \\
\hline Average number of lymph nodes per patient & $2.1(62 / 30)$ & $1.6(71 / 45)$ \\
\hline Long axis (mean) & $13 \mathrm{~mm}$ & $12 \mathrm{~mm}$ \\
\hline Short axis (mean) & $5 \mathrm{~mm}$ & $5 \mathrm{~mm}$ \\
\hline Largest mediastinal lymph node & & \\
\hline - Long axis (mean) & $17 \mathrm{~mm}$ & $16 \mathrm{~mm}$ \\
\hline - Short axis (mean) & $6 \mathrm{~mm}$ & $6 \mathrm{~mm}$ \\
\hline Central hyperechogenicity & $51.6 \%(32 / 62)$ & $29.6 \%(21 / 72)$ \\
\hline Round shape & $3(5 \%)$ & $5(7 \%)$ \\
\hline
\end{tabular}

mediastinal lymph node burden may influence the decision to perform FNA in a patient with detectable lymph nodes on EUS. Previous autopsy and CT studies had also shown a significant geographic variation in mediastinal lymph node prevalence [12-14]. The larger number of lymph nodes documented in those studies suggests that their prevalence could be underestimated by EUS [12-14]. However, due to the significant geographical variation documented for both techniques, this needs further comparison in the same population.

Although we found that patients with current or previous smoking habits and with relevant respiratory occupational exposure had a higher prevalence of mediastinal lymph nodes and there was a higher number of lymph nodes per patient, none of these clinical variables were independently associated with the presence of lymph nodes in logistic regression analysis. No differences were found in mediastinal lymph node prevalence or echo features between younger and older patients ( $<$ and $\geq 65$ years).

Most of the studies analyzing mediastinal lymph nodes by EUS were performed in patients with known malignant disease [5, 7-9]. In these studies, four echo features were classically associated with malignancy, ordered by decreasing sensitivity: hypoechoic echotexture, sharp edges, round shape and size (large-axis diameter) $>10 \mathrm{~mm}$ [8]. Pretest probability determines the specificity of these features. Catalano et al. [8]. showed that a patient with known esophageal neoplasia and detectable mediastinal lymph nodes on EUS, independently of its echo features, had a probability of $86 \%$ of being node-positive. In our study, in a population without malignant disease, prevalence of mediastinal lymph nodes was $72 \%$ and almost one-quarter of patients (24\%) were shown to have lymph nodes with a hypoechogenic homogeneous pattern. However, that is a very subjective feature, dependent on frequency and gain settings, and dependent on the region used for comparison, which was not defined in previous studies (we used the adjacent mediastinal fat as a reference). More than half of our patients (55\%) had mediastinal lymph nodes larger than $1 \mathrm{~cm}$. In the subcarinal region, $70 \%$ of the lymph nodes were larger than $1 \mathrm{~cm}$. Radiologists classically define a mediastinal lymph node with a short axis larger than $1 \mathrm{~cm}$ as adenopathy [19]. Short-axis diameter is more reproducible and improves the specificity of the lymph nodes size as a predictor for malignancy [19]. In our population, we found $6 \%$ of lymph nodes with a short axis $>1 \mathrm{~cm}-11 \%$ in the subcarinal space and none in the other mediastinal stations. 
Because $11 \%$ of the lymph nodes in the subcarinal space had a short-axis diameter $>1 \mathrm{~cm}$, even this criterion predictably has limited specificity for malignancy for this location in the analyzed population. Another relevant finding was that none of our patients simultaneously had 3 or 4 features that suggested lymph node malignancy, which supports the good specificity of these features when documented together in our population [8]. However, Buthani et al. showed that, even taken together, these echo features lack sensitivity, as they are present in only $25 \%$ of malignant lymph nodes [9].

FNA is superior to lymph node echo features in evaluating lymphadenopathies, particularly in the mediastinum [20]. However, that is an expensive procedure with a low but real risk of adverse events. Knowing the burden of mediastinal lymph nodes and its features in our population is crucial for appropriate selection of patients and targeting of lymph nodes for FNA. In the decision to perform FNA of a mediastinal lymph node, the clinical background and the pre-test probability of malignancy are major factors to take into account. A drawback of this study is that pathological analysis of the mediastinal lymph nodes was not performed. As the pretest probability for malignancy was very low in our population and all patients were asymptomatic, FNA of mediastinal lymph nodes was not performed. In addition, clinical evaluation and thoracic CT over the short term (within 3 months) did not show relevant findings in patients followed based on EUS findings.

\section{Conclusion}

In conclusion, this study documented a higher prevalence of mediastinal lymph nodes in a Southern European population than previously reported in Northern Europe, in patients with no evidence of oncologic disease [15]. This higher prevalence may negatively influence the specificity and positive predictive value for malignancy of mediastinal lymph node staging by EUS, which may be particularly relevant in esophageal and pulmonary cancer staging.

Competing interests

None

\section{References}

[1] Mountain CF, Dresler CM. Regional lymph node classification for lung cancer staging. Chest 1997; 111: 1718-1723

[2] Zielinski M, Rami-Porta R. Proposals for changes in the Mountain and Dresler mediastinal and pulmonary lymph node map. J Thorac Oncol 2007; 2: 3-6
[3] Kramer H, Groen HJM. Current concepts in the mediastinal lymph node staging of nonsmall cell lung cancer. Annals Surg 2003; 238: $180-188$

[4] Buxbaum JL, Eloubeidi MA. Endoscopic evaluation and treatment of esophageal cancer. Minerva Gastroenterol Dietol 2009; 55: 455-469

[5] Puli SR, Batapati Krishna Reddy J et al. How good is endoscopic ultrasound for TNM staging of gastric cancers? A meta-analysis and systematic review World J Gastroenterol 2008; 14: 4011 -4009

[6] Savides TJ, Perricone A. Impact of EUS-guided FNA of enlarged mediastinal lymph nodes on subsequent thoracic surgery rates. Gastrointest Endosc 2004; 60: $343-346$

[7] Annema JT, Versteegh MI, Veseliç M et al. Endoscopic ultrasound added to mediastinoscopy for preoperative staging of patients with lung cancer. JAMA 2005; 294: 931 - 936

[8] Catalano MF, Sivak MV, Rice T et al. Endosonographic features predictive of lymph node metastasis. Gastrointest Endosc 1994; 40: $442-446$

[9] Bhutani MS, Hawes RH, Hoffman B]. A comparison of the accuracy of echo features during endoscopic ultrasound (EUS) and EUS-guided fine-needle aspiration for diagnosis of malignant lymph node invasion. Gastrointest Endosc 1997; 45: 474-479

[10] Chen VK, Eloubeidi MA. Endoscopic ultrasound-guided fine needle aspiration is superior to lymph node echofeatures: a prospective evaluation of mediastinal and peri-intestinal lymphadenopathy. Am J Gastroenterol 2004; 99: 628-633

[11] Williams DB, Sahai AV, Aabakken L et al. Endoscopic ultrasound guided fine needle aspiration biopsy: a large single centre experience. Gut 1999; 44: $720-726$

[12] Glazer GM, Gross BH, Quint LE et al. Normal mediastinal lymph nodes: number and size according to American Thoracic Society mapping. Am J Roentgenol 1985; 144: 261-265

[13] Kiyono K, Sone S, Sakai F et al. The number and size of normal mediastinal lymph nodes: a postmortem study. Am J Roentgenol 1988; 150: $771-776$

[14] Herbella FA, Del Grande JC, Colleoni R. Anatomical analysis of the mediastinal lymph nodes of normal Brazilian subjects according to the classification of the Japanese Society for Diseases of the Esophagus. Surg Today 2003; 33: 249-253

[15] Kalaitzakis E, Sadik R, Doig L et al. Defining the lymph node burden in a Northern European population without malignancy: the potential effect of geography in determining a need for FNA? Dis Esophagus 2009; 22: 409-417

[16] Wiersema MJ, Hassig WM, Hawes RH et al. Mediastinal lymph node detection with endosonography. Gastrointest Endosc 1993; 39: 788 793

[17] World Health Organization. Global Tuberc Rep. WHO; 2015

[18] Wheat L], Azar MM, Bahr NC et al. Histoplasmosis. Infect Dis Clin N Am 2016; 30: $207-227$

[19] Sharma A, Fidias P, Hayman LA et al. Patterns of lymphadenopathy in thoracic malignancies. Radiographics 2014; 24: 419-424

[20] Chen VK, Eloubeidi MA. Endoscopic ultrasound-guided fine needle aspiration is superior to lymph node echofeatures: a prospective evaluation of mediastinal and peri-intestinal lymphadenopathy. Am J Gastroenterol 2004; 99: 628-633 